\title{
GRAFEMAS DE ALARGAMIENTO FONÉTICO-FONOLÓGICO DE LA LENGUA ALEMANA CON CONSIDERACIÓN DE LA ESTRUCTURA DE LA SÍLABA EN EL MARCO DE LA FONOLOGÍA SUPRASEGMENTAL
}

Ana Medina Reguera

\begin{abstract}
This paper discusses that the opposition of long and short vowels in Modern Standard German is not only segmental but prosodic, and how the segmental sequences are organized into prosodic constituents. The German orthographic lengthening signs, which are responsible for all phonological correlates of vowel quantity, are presented and the attention is then focused on the h-sign (in words like dehnen, sehen, Mühl, Schuh). A classification is made and their positions and functions are explored. Finally, the benefits of using these orthographic signs in the learning and teaching of German as a second language are argued.
\end{abstract}

Una de las tareas que se han propuesto las gramáticas y sobre todo las ortografías del alemán desde el siglo $\mathrm{XV}^{1}$ es indagar en cuanto a las relaciones que subyacen a la ordenación de letras y sonidos (grafemas y fonemas) en la cadena de segmentos de una forma grafemática determinada. Actualmente, esto sigue siendo tarea actual de lingüistas, sobre todo de fonólogos y en investigaciones en el marco de la ortografía. En las últimas décadas, se viene defendiendo en los países de lengua alemana una cierta independencia del sistema grafemático del alemán, que siempre había sido dependiente del sistema fonológico. Se están estableciendo indicios importantes, para hacer del sistema de escritura alemán un objeto de estudio independiente de la fonología.

A continuación se pretende demostrar cómo los signos de alargamiento fonéticofonológico de la lengua alemana pueden ser considerados como un ejemplo de las regularidades propias del sistema de escritura, que es el objeto de la grafemática. Para establecer dichas regularidades científicamente, es preciso establecer el inventario de los grafemas así como las relaciones de éstos con los fonemas correspondientes. Por ejemplo, la sucesión de los segmentos en <kalt> se corresponde en sus cuatro segmentos con la

\footnotetext{
${ }^{1}$ Las más destacadas son "Ortografia deutsch" de Fabian Frangt en 1531, la de Valentin Ickelsamer ("Die rechte weis aufs Kürzsist lesen zu lernen") en 1527, la de Hieronymus Freyer en 1675, o la gramática de Adelung en 1781 .
} 
sucesión de los fonemas $/ \mathrm{kalt} / 2$. Sin embargo, otras formas fonológicas como / $\mathrm{pi}$ :s/ o /bly:an/, no representan con relación de uno a uno las formas $<$ Spieß $>0<$ blühen $>$. Se quiere demostrar más abajo, que la existencia de la $<\mathrm{h}>$ en $<$ blühen $>$ no es algo arbitrario, sino que es necesaria porque llena el onset (margen inicial) de la segunda sílaba. Esto significa que la escritura de algunos grafemas está determinada por condiciones referentes a la estructura de la sílaba.

El alfabeto latino lo comparten muchos idiomas que no se asemejan necesariamente en gran medida en su estructura fónica. Este es uno de los motivos por los que el alfabeto latino ha ido evolucionando en algunos aspectos en los diferentes idiomas que de él hacen uso. El alfabeto del alemán ha evolucionado sobre todo en tres puntos importantes:

1 En el desarrollo de una nueva consonante (el grafema llamado "Eszet", $<\beta>$ )

2 En la creación de tres nuevas vocales a través de las tildes diacríticas (“Diakritika"): < $<>,<\ddot{0}>,<\ddot{u}>$

3 En la conglomeración de dos o más letras como unidades fijas: dígrafos y trígrafos $(<\mathrm{ch}>$, $<\mathrm{qu}>$, $<\mathrm{sch}>$, etc. $)$

Las reglas de correspondencia entre grafemas y fonemas (en los manuales de ortografía destinados a los usuarios de la lengua alemana recibe el nombre de Laut-BuchstabenKorrespondenz, es decir, ordenación de sonidos y letras) determinan cuáles son los segmentos de la lengua escrita que deben ser utilizados para configurar correctamente un sistema fiel a su representación fónico-fonológica. La existencia de las reglas de correspondencia entre la forma fonológica y la forma grafemática de una palabra teniendo en cuenta fonemas y grafemas se conoce como principio fonológico o fonográfico ("phonologisches / phonographisches Prinzip"). En lengua alemana podría esperarse que a cada grafema le correspondiera un fonema y a cada fonema un grafema. Los nuevos planteamientos prefieren sin embargo una variante menos restrictiva de esta correspondencia ideal (Dudengrammatik (1995:62), Neuregelung (1995:15), Bierwisch (1972), Primus (2000:2)): "Den Phoneme lassen sich regellhaft Segmente des Geschriebenen, nämlich Grapheme, zuordnen (phonographisches Prinzip)”.

Ambas variantes dejan de lado el plano suprasegmental de la fonología y de la grafemática, puesto que se basan únicamente en los segmentos. Como se pretende demostrar en este trabajo, siguiendo las relaciones que existen únicamente los segmentos, estas correspondencias pueden establecerse sólo en parte, puesto que tanto las formas fonológicas como las grafemáticas contienen información suprasegmental. Por lo tanto, pensamos que la distribución de fonemas y grafemas sólo puede ser analizada con ayuda del análisis de la estructura de la sílaba.

El libro de Chomsky y Halle de 1968, conocido mundialmente como SPE (The Sound Patterns of English) dio lugar a lo que se conoce con el nombre de Fonología no lineal, con

\footnotetext{
${ }^{2}$ La sucesión de grafemas se representa con los signos $\langle>$, la forma fonológica con las barras //, y la forma fonética con los corchetes [ ]. Cuando aparecen ejemplos de este modo ( $<$ Mutter $>$ ) se quiere dar importancia a la forma grafemática independientemente del significado, forma fonológica, función sintáctica etc.
} 
Grafemas de alargamiento fonético-fonológico de la lengua alemana...

sus dos planteamientos más importantes denominados Fonología autosegmental y Fonología métrica. La primera se ocupó principalmente de los segmentos y la segunda de unidades mayores como el pie y la mora. Sin embargo, en la actualidad la investigación en fonología allemana se centra en la prosodia o estructuras suprasegmentales, porque está resolviendo problemas de análisis en cuanto a la estructura de la sílaba, del acento, de la entonación, de la calidad y cantidad de los segmentos, etc. ${ }^{3}$

El presupuesto central de las teorías prosódicas se basa en el reconocimiento de estructuras jerárquicas más allá del plano de los segmentos (fonemas). Se crean varias capas o estratos (del inglés "tiers", alemán "Schichten") que se disponen unas encima de las otras.

La silbificación de las palabras (aquí nos referimos a palabras en el sentido de formas fonológicas y no a la silbificación de palabras a final de renglón) y la estructura de las sílabas del alemán son muy útiles para explicar fenómenos fonológicos del alemán como la cantidad y calidad de las vocales, y fenómenos grafemáticos como el doblamiento gráfico de las vocales y de las consonantes, o los grafemas de alargamiento entre otros, por lo que se tratará brevemente a continuación.

\section{LA ESTRUCTURA DE LA SÍLABA}

La sílaba es una unidad difícilmente definible en términos fonéticos, pero muy real para el hablante, ya que éste es mucho más consciente de estas unidades que de los segmentos por separado. Esto lo muestran entre otros investigaciones con analfabetos, que no presentaron problemas a la hora de dividir una cadena hablada en sílabas, pero no pudieron realizar la división de la cadena hablada en fonemas aislados (Ramers/Vater 1995:111).

La base fonética de la sílaba supone un movimiento rítmico creado por la sucesiva apertura y oclusión de los órganos articulatorios (1), y por la subida y bajada de la intensidad del sonido que producimos (escala de sonoridad, no en el sentido de oposición sonoro-sordo sino en el sentido de "Schallfülle"). La intensidad sube hasta llegar al núcleo de la sílaba (las vocales son por tanto los elementos más sonoros) y baja después del núcleo hasta el comienzo de la siguiente sílaba (2):

\footnotetext{
${ }^{3}$ Las investigaciones en fonología más importantes de los últimos veinte años tienen lugar en las universidades de München (cfr. Venemann (1986): Neuere Entwicklungen in der Phonologie. Berlin: Mouton de Gruyter), Wuppertal (cfr.Uhmann (1991): Fokusphonologie. Eine Analyse deutscher Intonationskonturen im Rahmen der nicht-linearen Phonologie, Tübingen: Niemeyer), Düsseldorf (cfr. Wiese (1996): The phonology of German. Oxford: Clarendon) y en Colonia (Eisenberg/Ramers/Vater (1992): Silbenphonologie des Deutschen. Tübingen: Narr).
} 
(1) Primus (2000:37)

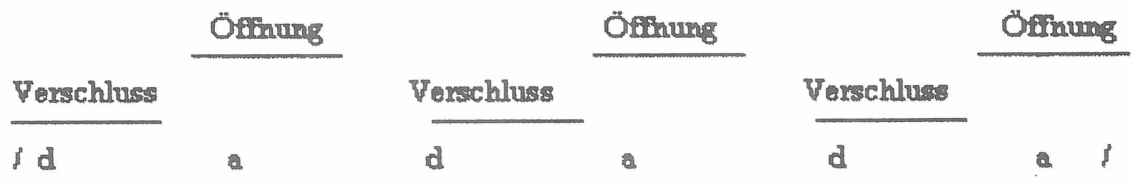

(2) Leners (1985:19)

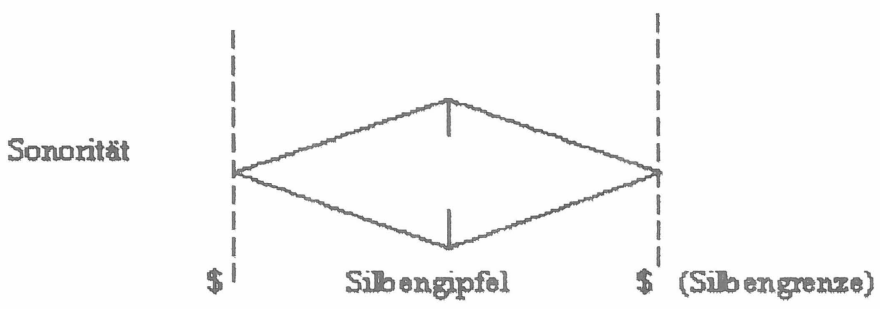

Una de las funciones de la sílaba es la de ser portadora de cualidades prosódicas tales como la entonación y el acento. Tras recientes investigaciones se ha demostrado que la sílaba también es la responsable de cantidad de la vocal y de la $S c h w a^{4}$. El modelo jerárquico de la estructura de la sílaba se denomina "Konstituentenstrukturmodel" y tiene varias variantes (de las que por motivos prácticos se tratará sólo una). Sus propiedades son:

1 Cada sílaba posee un núcleo.

2 Los dominios entre el núcleo y el límite izquierdo de sílaba es el onset (o "Silbenkopf" o "Anfangsrand").

3 Los dominios entre el núcleo (denominado en all. "Nukleus" o "Silbengipfel") y el margen izquierdo de la sílaba es llamado coda (también "Koda", "Endrand").

4 Un segmento puede ocupar dos posiciones en la estructura de la sílaba y dos posiciones pueden también ser ocupadas por un único segmento.

\footnotetext{
${ }^{4}$ La Schwa (también llamada Murmelvokal) es la vocal central que aparece únicamente en sílabas reducidas (Reduktionssilbe), es decir, aquellas sílabas que nunca van acentuadas. También se produce como alófono de otras vocales en sílabas no acentuadas. No existe ningún grafema específico para la Schwa, si bien ésta se suele corresponder con la <e $>$. La traducción al español de Alvárez (1995) es e-relajada, pero personalmente he optado por no usar este término.
} 
Grafemas de alargamiento fonético-fonológico de la lengua alemana...

5 En la "capa esquelética" (denominada "Skelettschicht") se sitúan las unidades suprasegmentales. La unidad C (originariamente del inglés "consonant") puede ser sin embargo ocupada por una vocal. Lo mismo ocurre con la unidad V (del ing. "vowel").

$6 \mathrm{La}$ mayor estructura posible de la sílaba en alemán es CCVCC (por ejemplo Krieg: /kri:k/). Todas las unidades que se encuentren fuera de este esquema (como ocurre con sílabas del tipo feilschst) se consideran extrasílbicas, dado que rompen la escala de sonoridad (tal como muestra el gráfico (2) ilustrado anteriormente).

7 Se distinguen entre dos tipos de sílabas con respecto a la acentuación: aquellas que son capaces de llevar el acento de la palabra ("betonbar") se denominan sílabas completas ("Vollsilben"), y aquellas que no pueden llevar nunca el acento de la palabra se denominan sílabas de reducción ("Reduktionssilben"). Éstas últimas son las sílabas cuyo núcleo es la Schwa.

8 El núcleo de las sílabas completas tiene dos posiciones que deben ser rellenadas obligatoriamente. Una vocal larga ocupa dos posiciones, un diptongo consta de una posición V (la primera vocal) y una posición C (la segunda vocal). Una vocal corta ocupa únicamente una posición en la capa de segmentos, por lo que debe seguirle una consonante para poder llenar la segunda posición del núcleo. La causa es la siguiente: una vocal larga, un diptongo y una secuencia de vocal corta + consonante son equivalentes fonotácticamente (en su duración). Esa es la razón por la que después de una vocal corta puede aparecer una consonante más que tras vocal larga o diptongo.

Ejemplos: (aus /aus/, Knie /kni:/, mit /mit/)
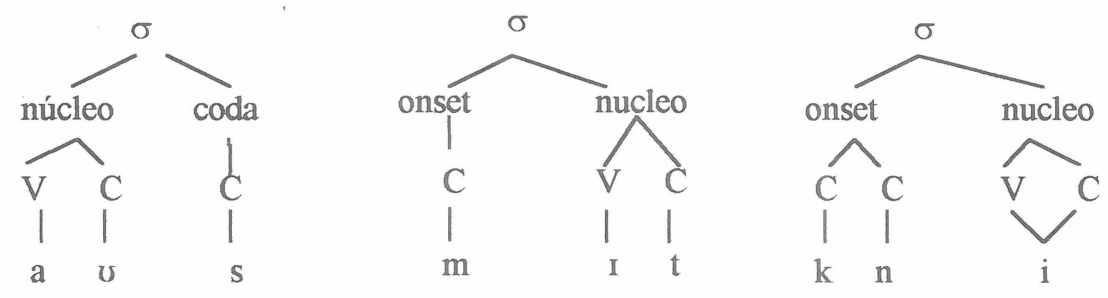

Una vocal corta ocupa únicamente una posición en la capa de segmentos, por lo que debe seguirle una consonante para poder llenar la segunda posición del núcleo. Esta regla (conocida como la condición del núcleo, en al. "Silbenkernbedingung") junto con la regla que exige la existencia del onset (que es ocupado normalmente por consonantes, en su defecto por el fonema oclusivo sonoro conocido como "glotal stop") en todas las sílabas "obliga" a aceptar también la existencia de consonantes ambisilábicas (también llamadas por algunos autores consonantes de articulación "Gelenkkonsonant"). 


\section{LOS SIGNOS DE ALARGAMIIENTO DEL ALEMÁN}

Los signos de alargamiento es uno de los capítulos más puntiagudos y complejos del ámbito ortográfico alemán Phonem-Graphem-Korrespondenz. La marca ortográfica para señalar la cantidad de las vocales aparece casi en su totalidad en palabras nativas y siempre en la raíz. A continuación se tratarán los cuatro mecanismos gráficos existentes en lengua alemana para mostrar el alargamiento fonológico:

\section{Grafema $<e>$ en correspondencia con el fonema /i:/}

El caso más regular es la $<\mathrm{e}>$ como alargamiento del fonema /i/. El grafema <ie $>$ aparece en representación del fonema /i:/ en sílaba acentuada. Algunos ejemplos son:

mieten, Liebe, nie, diskutieren, Knie, Biene, Spieß, die, sie, wie, Chemie, Ironie

Como se puede observar, las regularidades de distribución de estos grafemas son tres:

1) en sílaba abierta

2) al final de palabra

3) en el sufijo verbal -ieren.

Nunca aparece al principio de palabra y este hecho está relacionado con dos fenómenos: por un lado, una razón plausible parece ser que la $<\mathrm{i}\rangle$ precedida de $\langle\mathrm{e}\rangle$ evolucionó $\mathrm{a}\langle\mathrm{j}\rangle$, en escasos ejemplos como jemand, jeder, o jedoch. Por otro lado, el fonema vocálico para la /i:/ larga sólo se encuentra acompañado de una $<\mathrm{h}>$ de alargamiento en los pronombres ihnen, ihre, ihm, ihn, ihr. Han sido varios los intentos de justificar las formas de estos pronombres. En algunos autores puede leerse como ejemplo del principio estético ortográfico. La versión más aceptada es sin embargo la de evitar los homógrafos con las formas grafemáticas <im> e <in>, mientras que las demás estarían formadas analógicamente. Otra posibilidad es que el grafema $<\mathrm{h}>$ realice simplemente la función de marcar en la lectura el rasgo de cantidad y dar así "peso" grafemático a dichas formas.

El alargamiento del fonema /i/ por medio del grafema vocálico <e $>$ (también llamado Dehnungs-e) tiene una explicación histórica a través del la monoptongación del diptongo del alemán medio alto /iə/, que pasó al alemán nuevo como /i:/ por ejemplo:

mhd. [liəbø] $\rightarrow$ nhd. [li:bø]

mhd. $<$ liebe $>\rightarrow$ nhd. $<$ Liebe $>$

El fonema / / enmudeció en su pronunciación pero el grafema $<\mathrm{e}>$ permaneció en la escritura, y progresivamente, empezó a reconocerse este grafema como signo de alargamiento del fonema anterior. Este es un hecho curiosísimo y uno de los motivos para pensar que la grafemática tiene su propio modus operandi, dado que se encuentran actualmente incontables casos que no pueden ser explicados históricamente a través de la monoptongación. Augst (1974:18) ofrece el siguiente ejemplo:

$$
\begin{aligned}
& \text { mhd. [risə] } \rightarrow \text { nhd. [ri:sə] } \\
& \text { mhd. }<\text { rise }>\rightarrow \text { nhd. }<\text { Riese }>
\end{aligned}
$$


Grafemas de alargamiento fonético-fonológico de la lengua alemana...

Esta regla por la que debía señalizarse la /i:/ con el grafema $<\mathrm{e}>$ se siguió prácticamente a rajatabla. Incluso el sufijo [i:ron] que puede encontrarse en el Libro de Reglas Prusianas ${ }^{5}$ como <-iren>, apareció posteriormente como <ieren>.

Esta es una prueba de que las regularidades ortográficas no están siempre motivadas diacrónicamente. El dígrafo <ie> es tan frecuente que representa el caso normal, frente al fonema vocálico largo /i:/ realizado grafemáticamente con <i > (Diva, Iris, Krise, Ventil) que representa el caso marcado para palabras extranjeras. Esto puede ser un problema de tipo didáctico dado que dentro del corpus de estas palabras extranjeras se encuentran palabras del tipo Bibel, Brise, Tiger, Alpelsine, Familie, Musik o Cousine que pertenecen al vocabulario cotidiano y frecuente del alemán. Es interesante también que en estos casos hay diferencias regionales, fundadas seguramente en la forma ortográfica, y así encontramos alternaciones de los fonemas /i:/ - /I/ como muestran los siguientes ejemplos:

$$
\begin{array}{ll}
\text { Liter: } & \text { ['li:te] o ['lite] } \\
\text { gibt: } & \text { ['gi:pt] o ['grpt], } \\
\text { Fabrik: } & \text { [fa'bri:k] o [fa'bruk] } \\
\text { Musik: } & \text { ['muzik] o [mu'zi:k]. }
\end{array}
$$

\section{Reduplicación de grafemas vocálicos}

De todos los grafemas vocálicos aquellos que se doblan son $\langle\mathrm{a}\rangle,\langle\mathrm{e}\rangle, \mathrm{y}\langle 0\rangle$. El doblamiento de las vocales $\langle a a\rangle,\langle 00\rangle$ y $\langle$ ee $>$ no es muy frecuente en lengua alemana, apareciendo en aproximadamente 200 casos (Maas 2000:449).

Los grafemas $<\mathrm{i}>\mathrm{e}<\mathrm{u}>$ no se doblan porque esto llevaría a irritaciones en la lectura $\left(*<\mathrm{ii}>,{ }^{*}<\mathrm{uu}>\right.$ ) así como confusiones con los grafemas $<\mathrm{n}>\mathrm{y}<\mathrm{m}>$. Por este motivo tampoco se doblan los grafemas con diacríticos. Aparece entonces un único grafema: así, de formas grafemáticas como $<$ Haar $>$ no obtenemos la forma grafemática $*<$ Häärchen $>$ sino $<$ Härchen $>$, de $<$ Boot $>$, no se deriva $*<$ Böötchen $>$ sino $<$ Bötchen $>$. Los grafemas vocálicos geminados pueden aparecen en posición intermedia o final. Algunos ejemplos en posición final son:

Schnee, See, Allee, Kaffee.

Antes de consonante la duplicación de los grafemas vocálicos suele aparecer antes de $<\mathrm{r}>,<\mathrm{l}>, 0<\mathrm{l}>$ :

Haar, Paar, Maar, Saal, Aal, Staat, Maat, Saat

Beere, Heer, leer, Meer, Speer, Teer, Seele, scheel, Reet, Beet

\footnotetext{
5 Allí estaban recogidas las reglas de ortografía escolares en las que se basó Konrad Duden para realizar su diccionario "Die deutsche Rechtschreibung" en 1880.

${ }^{6}$ Utz Maas (2000:498) recuerda que hay casos especialmente interesantes cuando se didactiza este grafema, ya que existen casos dificiles como wider vs. wieder, Lied vs. Lid, Fieber vs. Fiber y otros de otro tipo como vielleicht [fi' larçt] o Beispiel ['bar.Jpil] en los que la <ie $>$ no se corresponde con la /i:/ tensa sino con la tenue.
} 
Moor, Boot

Sin embargo, la duplicación también existe precediendo a otras consonantes como en Moos, Aas, doof, Waage o Koog.

La posición antes de los grafemas $<\mathrm{r}>\mathrm{y}<\mathrm{l}>$ tiene relación con el contexto del segundo tipo de $<\mathrm{h}>$ de alargamiento. La $<\mathrm{t}>\mathrm{y}<\mathrm{s}>$ por otro lado son los grafemas que aparecen con más frecuencia cuando el margen derecho de la sílaba es complejo (en las codas de más de un segmento). Con la reduplicación de estos grafemas se marca la calidad y cantidad de los fonemas vocálicos que aparecen antes de los grafemas consonánticos correspondientes con codas complejas y que por tanto se relacionan normalmente con la vocal tenue.

Existe también un subgrupo con características especiales. Se trata del grafema <ee $>$ en sílaba abierta. Es un subgrupo heterogéneo porque hay por un lado palabras nativas (Fee, Schee, See, Lee, Klee) por otro préstamos, sobre todo franceses (Allee, Armee, Kaffee, Kaktee, Klischee, Komitee, Livree, Orchidee, Puree, Tournee) y un tercer tipo son palabras extranjeras que han adaptado la grafía con la nueva reforma (préstamos del francés que se escribían antes con <é>): Dublee, Exposee, Kommunikee, Varietee

Todas las formas que abarcan este grupo de palabras con doble grafema vocálico poseen la misma característica fonológica: La segunda vocal no se realiza fonológicamente, sino que marca la cantidad de la primera vocal, que es realizada fonéticamente larga. En la estructura suprasegmental de estas sílabas, la segunda vocal rellena la segunda posición del núcleo.

A continuación se compararán los siguientes pares mínimos:

die Armee / die Arme

die Ideen / die Iden (des März)

die Allee / alle

A la vista de estos ejemplos se puede apreciar que las dobles vocales no funcionan únicamente como signo de alargamiento, sino que también muestran el estatus de la vocal como "vocal completa" ("Vollvokal"). Esto presupone la división de las vocales en "Vollvokal" y "vocal de reducción" ("Reduktionsvokal"). Las primeras son aquellas que pueden, es decir, una de sus características es la de tener la capacidad de llevar el acento de la palabra, y las segundas, llamadas vocales de reducción son siempre átonas, uno de sus rasgos fonológicos es la incapacidad de llevar el acento. En alemán esta vocal es la $S c h w a$. Dado que el grafema $<\mathrm{e}>$ al final de palabra está siempre relacionada con el fonema /a/ es apropiado doblarla para no dejar duda de que la vocal es tónica. Además, como apunta Primus (2000:14): "Demnach können in Reduktionssilben keine Dehnungszeichen vorkommen, weil diesen Silben die für Dehnungszeichen notwendigen nukleare C-Position fehlt.“

La estrecha relación entre el alargamiento y el acento lo muestran los verbos terminados en <-ieren> como rasieren, telefonieren, kapieren etc (recogidos en $\S 11$ ). Prácticamente no existe ningún caso en el que se produzca doblamiento del grafema vocálico en sílabas no acentuadas. Las excepciones son 'Kaffee, 'Kanapee, que vienen 
Grafemas de alargamiento fonético-fonológico de la lengua alemana...

del francés, pero incluso en este caso la grafía tiene una función interesante, aligerando un poco la carga funcional del grafema $<\mathrm{e}\rangle$, que se corresponde all final de palabra con una $S c h w a$. Así puede diferenciarse el fonema breve y tenue en Kaff/e/ de la $S c h w a$ a final de palabra en formas grafemáticas como $<$ Hase $>,<$ Name $>0<$ bitte $>$.

\section{Grafema $<\mathrm{h}>$ de alargamiento (Dehnungs- $h$ )}

$\mathrm{La}<\mathrm{h}>$ representa el signo de alargamiento prototípico, aunque la variedad de posiciones en las que aparece complica su análisis y aumenta sus funciones. Partimos del grafema que no tiene correspondencia con el fonema fricativo glotal (o laríngeo), es decir, no precedente a la vocal. El fonema fricativo glotal $/ \mathrm{h} /$ tiene además una distribución distinta, pues aparece siempre el onset. El grafema de alargamiento puede aparecer en el onset o en la coda de las sílabas.

Se habla en estos casos también de una "h muda" (stummes $h$ ), ya que, como se hemos dicho no tiene correspondencia con ningún fonema. ${ }^{\top}$ Distinguiremos entre tres tipos distintos:

\section{A. Primer tipo de $<\mathrm{h}>$ : inicial de sílaba}

$\mathrm{El}$ primer tipo se conoce como silbentrennendes, silbenöffnendes o silbeninitiales $<\mathrm{h}>$, dado que se la caracteriza como marcador de formas bisílabas además de como alargador de la vocal precedente. Su situación es entre dos sílabas, de las cuales la primera es acentuada y abierta y la segunda no acentuada y "desnuda" (nackt, es decir, sin onset). Entre ambas sílabas aparece regularmente una $<\mathrm{h}>$. Son palabras del tipo:

Ruhe, Brühe, fliehen, früher, Ehe, stehen, Flöhe, nähen, nahes, drohen, fähig

Como puede observarse en los ejemplos, la gran mayoría de estas palabras poseen el fonema Schwa $(/ / /)$ en la segunda sílaba, esto se debe a que las sílabas con Schwa constituyen las sílabas no acentuadas por excelencia en alemán. Sin embargo esto no ocurre necesariamente como muestran los ejemplos fähig o Drohung (sufijos -ig y -ung respectivamente).

A continuación se han buscado las regularidades grafotácticas (de aparición) de este grafema:

Esta $<\mathrm{h}>$ aparece tras todos los grafemas vocálicos con excepción de $<\mathrm{i}>8$. La $<\mathrm{h}>$ no puede aparecer detrás de $<\mathrm{i}>$ porque en muy pocos casos la /i/ es larga y va acentuada (a principio de palabra en la última edición del Rechtschreibduden sólo se encuentran Ibis, Igel, Iden, Iglu, Iktus, Iler, Ilex, Ire, Iris, Isegrim, Isolinie, Itaker, Item, Iwan y aquellas que iban acentuadas evolucionaron en algún momento a $<j>$ (iemand $>j$ jemand). Este es seguramente el motivo por el que no existe $*<$ ih $>$ pero sí $<$ ieh $>$. También aparece tras el diptongo <ei > como muestran los ejemplos Reihe, verzeihen, Geweihe, weihen, leihen, gedeihen, Weiher aunque no siempre, como muestran los ejemplos Eier, Zweier, Schleie,

\footnotetext{
${ }^{7}$ Está bastante superado, aunque como lamenta Eisenberg: "Leider halten manche Didaktiker nach wie vor die Überzeugung hoch, es gebe heir etwas zu hören"

${ }^{8}$ Excluyendo las formas pronominales ihr, ihn, ihm, ihre, etc. de las que se ha hablado antes.
} 
freies, Feier, schneien, schreien, speien. Estos últimos se consideran como excepciones (o como el caso marcado) ya que en leihen, verzeihen, etc. la $<\mathrm{h}>$ permanece en todas las formas (lieh, verziehen) mientras que en schreien y speien el participio es geschrien y gespien (antes de la nueva ortografía estaba permitido escribir geschrieen y gespieen). También antes de la reforma ortográfica de 1996 existía la $<\mathrm{h}>$ inicial de sílaba antes del diptongo <au $>$ pero únicamente en los casos $<$ rauh $>$ y $<$ rauhen $>$, ahora reformados en $<$ rau $>$, $<$ rauen $>$ al igual que sus análogos $<$ blau $>$ y $<$ klauen $>$.

Una de las funciones de este grafema es estética, en tanto que evita el encuentro de varias vocales sobre todo de $<\mathrm{i}>$ y de $<\mathrm{e}>$ en formas como ${ }^{*}<$ zieen $>$, ${ }^{*}<$ ween $>$, que quedan bloqueadas por la $<\mathrm{h}>$ en $<$ ziehen $>$, $<$ wehen $>$.

Otra de las funciones es la de marcar en la lectura la frontera entre las dos sílabas, y le atribuye fácilmente a las dos sílabas su vocal correspondiente. Eisenberg (1998:300) apunta que además la $<\mathrm{h}>$ contribuye a la clasificación de las sílabas, y compara formas como $<$ rotes $>$, $<$ spä̈tes $>,<$ Rute $>$, con $<$ rohes $>$, $<$ näher $>$, $<$ Ruhe $>$. La constitución de la segunda sílaba es la misma en ambos grupos.

Fonólogos como Wiese, Ramers o Primus, ven una función fonológica importante, ya que la $<\mathrm{h}>$ llena el onset de la segunda sílaba, igual que lo hacen la $\langle\mathrm{t}\rangle$ en $<$ rote $>0<$ Rute $>$ que tienen también un onset simple. En la forma fonológica de $<$ Ruhe $>0<$ fähig $>$, el onset está vacio /ru:ə/, pero en la forma grafemática la $<\mathrm{h}>$ hace de marcador de separación de sílaba por un lado, dado que el onset es el primer elemento de la misma, y de indicador de cantidad de la vocal por otro, pues como en casi todas las sílabas abiertas la vocal es larga, ocupando por tanto las dos posiciones correspondientes del núcleo. A continuación se muestra un ejemplo de la representación suprasegmental de la forma grafemática de $<$ Ruhe $>$ :

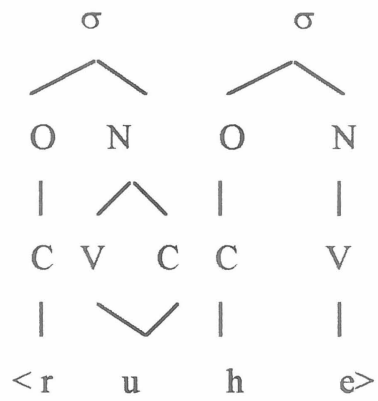

Por último, existen algunas formas monosílabas que pueden incluirse dentro de este grupo y que suelen explicarse siguiendo el principio morfemático. Algunos ejemplos son:

früh, Kuh, ruh, Schuh, zäh, nah, Reh, weh, Floh, Stroh

Cuando estas formas se flexionan (früher, Floh, Kühe, ruhen, Schuhen, zähen, etc.) la $<\mathrm{h}>$ entresilábica es perfectamente regular. Otro intento de regularización ofrecen Maas o Primus, que al diferenciar entre fonologische Silbengewicht y graphematische 
Grafemas de alargamiento fonético-fonológico de la lengua alemana...

Silbengewicht, le otorgan a la $<\mathrm{h}>$ en estas palabras la función de optimizar la sílaba añadiéndole un poco más de "peso" con el grafema $<\mathrm{h}>$. Dado que Eisenberg se acoge con mucha frecuencia al principio morfemático, me parece conveniente tener en cuenta también la teoría del "peso grafemático", sobre todo observando las interjecciones del tipo $a h, b a h$, oh, uh, Buh, que no pueden ser ampliadas a formas bisílabas.

B. Segundo tipo de $<\mathrm{h}>$ : precedente de sonorantes

Ya Adelung (1788: 221) observó que existe en lengua alemana un caso sistemático, el de la $<\mathrm{h}>$ antes de sonorantes (se llama sonorantes al grupo comprendido por las líquidas y nasales, es decir $/ \mathrm{r} /, / 1 /, / \mathrm{n} / \mathrm{y} / \mathrm{m} /$ ). La primera diferencia que se observa es que, si en el tipo anterior el fenómeno sólo afectaba a palabras bisílabas, en este tipo también los monosílabos pueden llevar una $<\mathrm{h}>$ de alargamiento. En este caso la primera sílaba no es abierta como en el caso anterior sino cerrada, y la segunda sílaba (si la hay) comienza con una sonorante y nunca está acentuada. La $<\mathrm{h}>$ es el último elemento de la primera sílaba (la coda). Ejemplos:

Bahre, Sahne, Huhn, Wahn, kühn, Sühne, Lahm, kühn, Fuhre, Kohle, Bohne, Rahmen, Lehrer, dehnen, Hehler, stehlen

Este caso no es tan sistemático como el anterior, dado que la $<\mathrm{h}>$ aparece en aproximadamente un 50 por ciento de los casos. En la otra mitad no aparece como muestran los ejemplos:

Ware, Schere, Schale, Schule, Krone, Blume

También en monosílabos alternan las dos posibilidades:

Tal, bar, Tür, Mal, Qual, schwer, Tor, Flor, Schur

frente a:

Hahn, hohl, Jahr, Ohr, Uhr, Sohn, zehn, Kohl, Maht, Draht, Naht, Pfahl, Stahl, Brahm, Ruhm

Las sonorantes son los elementos más sonoros después de las vocales y forman con ellas un bloque coherente de sonidos en el centro de la sílaba. En los márgenes de las sílabas son los elementos que más cerca están de las vocales. Y al contrario, en la coda si a la vocal nuclear le sigue una sonorante, la coda es seguramente compleja en monosílabos, como en $<$ hart $>$, <ernst $>$, $<$ kalt $>,<$ Gans $>$, y en bisílabos la primera sílaba es cerrada, como en $<$ rannte $>$, Ambas cosas tienen que ver con que la vocal es normalmente tenue y corta si le sigue un sonorante. En este sentido la $<\mathrm{h}>$ es una ayuda para la lectura, pues muestra el caso infrecuente en el que la vocal que le precede es larga y tensa. La tendencia es que la $<\mathrm{h}>$ es más probable, cuanto más ligero es el onset, $<\mathrm{k}$ khl $>,<\mathrm{küh} n>$, $<\mathrm{kühl}>$, y menos probable mientras más complejo sea $<$ Schwan $>,<$ Schnur $><$ klar $>$, $<$ schwer $>$, $<$ Strom $>$.

\section{Tercer tipo de $<\mathrm{h}>$ : anterior al grafema <ie $>$}

Nos encontramos formas con lo que podríamos llamar "doble alargamiento" ( $<\mathrm{ie}>+$ $<\mathrm{h}>$ ). Algunos ejemplos son:

fliehen, ziehen, Vieh, empfiehlt, sieht, verziehen, geschieht 
Después de $<\mathrm{ie}>$ hay una $<\mathrm{h}>$ en los casos que están morfológicamente determinados, es decir, la $<\mathrm{h}>$ se hereda de la raíz del infinitivo, como en $<$ befiehlt $>$ de $<$ befehlen $>$, $<$ empfiehlt $>$ de $<$ empfehlen $>0<$ sieht $>$ de $<$ sehen $>$. En estos casos no existe condición alguna para alargar la vocal, dado que ya contamos con la $<\mathrm{e}>\left({ }^{*}<\right.$ siet $>$ hubiera bastado). En este caso puede hablarse de escrituras morfológicas ("morphologische Schreibungen"), esto significa que la ortografía se rige por el principio morfológico y no por el fonológico. La escritura pertenece fiel a los morfemas, aunque su motivación fonológica no sea correcta (por ejemplo en fonología /tak/ - /ta:gə/, pero en la escritura Tag-Tage).

Maas ha recogido las formas del antiguo Duden (antes de la reforma ortográfica) y ha encontrado 421 formas que contienen la secuencia $<\mathrm{ieh}>$. Resulta interesante que de todos los casos pueden extraerse únicamente doce morfemas diferentes:

-zieh, -Vieh, -flieh, -wieher, -befiehl, -empfiehl, -lieh, -rieh, -dieh, -schieh, -sieh, -stiehl

Entre estos morfemas hay cuatro que sólo cuentan con una única: -zieh (verziehen), rieh (geriehen), -dieh (gedieht), -schieh (geschieht).

\section{Conclusión}

Los signos gráfico de alargamiento fonético-fonológico presentan una estructura regular, distribuciones regulares, y sus excepciones no son numerosas y pertenecen en gran parte al corpus de extranjerismos. Con ayuda de la estructura de la sílaba, que ha sido presentada e ilustrada a través de teorías fonológicas suprasegmentales, se ha mostrado que los grafemas no han permanecido en la escritura arbitrariamente, sino para cumplir requisitos determinados y desempeñar funciones concretas en los ámbitos fonológicos y grafemáticos. Los signos de alargamiento deben ser didactizados como tales porque suponen una gran ayuda en cuanto al aprendizaje de la pronunciación del alemán (y por supuesto en el aprendizaje de la fonología alemana). Esto es importante teniendo en cuenta que la duración de los fonemas vocálicos en lengua española llega a alcanzar como máximo las 15 centésimas de segundo mientras que en alemán las vocales largas tienen una duración aproximada de $45 \mathrm{cs}$. de segundo. La correcta pronunciación de la cantidad de la vocal alemana debe ser en mi opinión uno de los capítulos importantes en la enseñanza de su pronunciación, sobre todo considerando, como se acaba de mostrar, las facilidades que existen de reconocer las vocales largas a través de los signos de alargamiento arriba presentados.

\section{BibLIOGRAFÍA:}

Augst, G. (1985): Regeln zur deutschen Rechtschreibung vom 1. Januar 2001. Entwurf einer neuen Verordnung zur Bereinigung der Laut-Buchstaben-Beziehung, Frankfurt

Augst (1985): "Dehnungs -hund Geminate in der graphematischen Struktur". Augst: Graphematik und Orthographie. págs. 112-121

Augst/Stock (1997): "Laut-Buchstaben-Zuordnung“. Augst et al., págs. 113-134

Becker, Thomas (1996): "Zur Repräsentation der Vokallänge". En: Zeitschrift für Sprachwissenschaft 15, págs. 3-21 
Grafemas de alargamiento fonético-fonológico de la lengua alemana...

Butt,M./Eisenberg P. (1990):"SChreibsilbe und Sprechsilbe“. Stetter, C (Ed.) (1990): Zu einer Theorie der Orthographie. Tübingen, págs. 33-64

Chomsky, Noam/Halle, Morris (1968): The Sound Pattern of English, New York, Harper \& Row

Duden (1995): Grammatik der deutschen Gegenwartssprache. Mannheim/ Leipzig/ Wien/ Zürich: Dudenverlag (Duden 4)

Duden (1996): Rechtschreibung der deutschen Sprache. Mannheim/ Leipzig/ Wien/ Zürich: Dudenverlag (Duden 1)

Eisenberg, Peter (1988): Graphemtheorie und phonologischens Prinzip. Vom Sinn eines autonomen Graphembegriffs

Eisenberg, Peter (1988): "Die Grapheme des Deutschen und ihre Beziehung zu den Phonemen“. Germanistische Linguistik 93/94, págs.346-360

Eisenberg, Peter (1989): "Die Schreibsilbe im Deutschen". En: Eisenberg, P./Günther, H. (Edd.) págs. 57-84

Eisenberg, Peter (1991): "Syllabische Struktur und Wortakzent. Prinzipien der Prosodik deutscher Wörter“. Zeitschrift für Sprachwissenschaft 10, págs. 37-64

Eisenberg, Peter (1995): "Der Buchstabe und die Schriftstruktur des Wortes". DudenGramatik, págs. 56-84

Eisenberg, Peter (1997): "Die besondere Kennzeichnung der kurzen Vokale - Vergleich und Bewertung der Neuregelung". Augst/Blüml/Nerius/Sitta, Edd, Zur Neuregelung der deutschen Orthographie. Begründung und Kritik. Tübingen.

Eisenberg, Peter (1999): Vokalbezeichnung als Problem. Linguistische Berichte 179, págs. 343-349.

Gierich, Heinz (1985): Metrical phonology and phonological structure. German and English. Cambridge: Cambridge University Press

Kohrt, Manfred (1985): Problemgeschichte des Graphembegriffs und des frühen Phonembegriffs, Tübingen, Niemeyer

Kohrt, Manfred (1985b): "Die wundersamen Mären vom "silbentrennenden $h$ ". Eisenber/Günther, págs. 179-228

Maas, Utz (1989): "Dehnung und Schärfung in der deutschen Orthographie". En: Eisenberg/Günther, págs. 229-250

Maas, Utz (1995): "Graphem-Phonem-Korrespondenz und die Vermeidung der Silbenstruktur“. Sprachwissenschaft 20, págs. 207-221

Maas, Utz (1997): "Orthographische Regularitäten, Regeln und ihre Deregulierung". Am Beispiel der Dehnungszeichen im Deutschen. Augst et. al. (Hr.), págs. 337-364 
Neef, Martín (2000): "Die Distribution des [h] im Deutschen: Schriftaussprache und Phonologie“. Convivium, págs. 271-286.

Primus, Beatrice (1999): "Suprasegmentale Graphematik und Phonologie: Die Dehnungszeichen im Deutschen". Linguistische Berichte 181

Ramers, Karl-Heinz (1992): "Ambisilbische Konsonanten im Deutschen". En: P. Eisenberg, K.H. Ramers, H. Vater, Edd., Silbenphonologie des Deutschen. Tübingen, Narr (SdG 42), págs. 246-283.

Ramers, Karl-Heinz (1998): Einführung in die Phonologie, Fink, München.

Ramers, Karl-Heinz (1999a): "Vokalquantität als orthographisches Problem: Zur Funktion der Doppelkonsonanzschreibung im Deutschen", Linguistische Berichte 177, págs. 52-64.

Ramers, Karl-Heinz/ Vater, Heinz (1995): Einführung in die Phonologie, Hürth, Gabel Verlag

Vater, Heinz (1992): "Zum Silben-Nukleus im Deutschen". En: P. Eisenberg, K.H. Ramers, H. Vater, Ed., Silbenphonologie des Deutschen, Tübingen, Narr (SdG 42), págs. 246-283.

Vater, Heinz (1998): "Zur Silbenstruktur im Deutschen." En: B.J. Kröger et al., Edd., Festschrift Georg Heike (Forum Phoneticum 66). Frankfurt am Main, págs. 137-149.

Wiese, Richard (1987): "Laut, Schrift und das Lexikon". En: Deutsche Sprache 15, págs. $318-35$

Wiese, Richard (1996): The Phonology of German, Oxford, Clarendon 